\title{
Performance of irradiated and crosslinked ethylene vinyl acetate/waste tire dust blend
}

\begin{abstract}
The effect of irradiation on a 90/10 ethylene vinyl acetate/waste tire dust (EVA/WTD) blend was studied in the presence of $4 \mathrm{phr}$ trimethylolpropane triacrylate (TMPTA) or tripropyleneglycol diacrylate (TPGDA). The blend was irradiated using a 3.0-MeV electron beam machine at $0,50,100,150$, and $200 \mathrm{kGy}$ irradiation doses. The properties studied include gel content, tensile properties, hardness, morphology, and dynamic mechanical properties. The result revealed that the increment in irradiation doses will increase the percentage of gel content. The gel content further increased with the introduction of TMPTA and TPGDA. This is attributed to the increase in crosslink density. Tensile properties of EVA/WTD blend increase with the introduction of irradiation except for elongation at break. The tensile-fractured surface of EVA/WTD blends showed ductile type of failure upon irradiation of the blends in the presence of crosslinking agent. One glass transition temperature obtained from dynamic mechanical analysis for 90/10 EVA/WTD blend after irradiation indicates that compatibility between EVA and WTD phases increased.
\end{abstract}

Keyword: Crosslinking; Electron microscopy; Irradiation effect; Mechanical properties; Polymer blends 